PROCEEDINGS OF THE

AMERICAN MATHEMATICAL SOCIETY

Volume 126, Number 10, October 1998, Pages 3055-3059

S 0002-9939(98)04538-9

\title{
THE RUSSO-DYE THEOREM IN NEST ALGEBRAS
}

\author{
KENNETH R. DAVIDSON
}

(Communicated by Palle E. T. Jorgensen)

\begin{abstract}
It is shown that the convex hull of the unitary elements of a nest algebra contains the whole unit ball if and only if both $0_{+}$and $I_{-}^{\perp}$ are either zero or infinite rank.
\end{abstract}

In [1], Anoussis and Katsoulis proved the surprising fact that for any nest with no finite atoms, every strict contraction in the nest algebra is the average of unitary elements in the algebra. This was quite remarkable because nonselfadjoint algebras such as nest algebras do not obviously have an abundance of unitary elements. It is also the case that triangular algebras have many extreme points in the unit ball which are not unitaries or even partial isometries [5]. This also makes it surprising that the convex hull of the set of unitary elements would suffice to recover the whole ball. In this note, we determine precisely which nests have this property.

Results of this kind are well-known for $\mathrm{C}^{*}$-algebras. Russo and Dye [6] established that the closed convex hull of the unitary group of any $\mathrm{C}^{*}$-algebra is the closed unit ball. A number of refinements on this theorem led to the KadisonPedersen result [3] that every strict contraction in a $\mathrm{C}^{*}$-algebra is the average of a finite number of unitary elements.

On the other hand, the simplest model for a nest algebra is the algebra $\mathcal{T}_{n}$ of all $n \times n$ upper triangular matrices. The only unitary elements in this algebra are diagonal. Thus the convex hull of the unitary elements is small relative to the whole unit ball of $\mathcal{T}_{n}$. A similar obstruction occurs in any nest $\mathcal{N}$ with an initial finite rank atom $E$. For then any unitary element $U$ in $\mathcal{T}(\mathcal{N})$ will have $U E=E U E$ as an isometry of $E \mathcal{H}$ into, whence onto, itself. Therefore $E U E^{\perp}=0$. The same obstruction obtains if there is a terminal finite rank atom. We show that these are the only obstructions.

Naturally the problem is to construct unitary elements in the nest algebra that approximate a given operator appropriately. Anoussis and Katsoulis [1] observed that any strict contraction is the average of strict contractions of the form $P^{\perp} A P^{\perp}$, where $P$ is a diagonal projection with support $I$. Their techniques required $P$ to be a sum of infinite rank intervals of the nest with special properties. Our improvement is to make do with sums of finite rank projections.

We refer to [2] for notation and background on nest algebras. Let $\mathcal{N}$ be a nest and let $\mathcal{T}(\mathcal{N})$ be the associated nest algebra. We will need the notation

$$
N_{+}=\inf \{M \in \mathcal{N}: M>N\} \quad \text { and } \quad N_{-}=\sup \{M \in \mathcal{N}: M<N\} .
$$

Received by the editors March 17, 1997.

1991 Mathematics Subject Classification. Primary 47D25.

The author was partially supported by an NSERC grant and a Killam Research Fellowship. 
An interval of $\mathcal{N}$ is a projection $E=N_{2}-N_{1}$, where $N_{1}<N_{2}$ belong to $\mathcal{N}$. The $\operatorname{support} \operatorname{supp}(e)$ in $\mathcal{N}$ of a vector $e$ is the smallest interval $E$ such that $E e=e$. Similarly the support of a projection in the diagonal algebra $\mathcal{N}^{\prime}$ is the smallest interval supporting every vector its range. If $E$ and $F$ are two intervals, say that $E \preceq F$ if $E \mathcal{T}(\mathcal{N}) F=E \mathcal{B}(\mathcal{H}) F$. Note that $N_{+} \preceq N^{\perp}$.

The notation $x y^{*}$ denotes the rank one operator $x y^{*}(v)=(v, y) x$ for $v \in \mathcal{H}$.

We begin with an easy result constructing useful partial isometries in $\mathcal{T}(\mathcal{N})$.

Lemma 1. Let $Q$ be a diagonal projection in $\mathcal{T}(\mathcal{N})$; and let $f_{k}$ be an orthonormal set such that infinitely many $f_{k}$ 's belong to $N$ for every $N>0$ in $\mathcal{N}$. Then there is a partial isometry $S$ in $\mathcal{T}(\mathcal{N})$ such that $S^{*} S=Q$ and its range is spanned by a subset of the $f_{k}$ 's.

Similarly, if $P$ is a diagonal projection in $\mathcal{T}(\mathcal{N})$ and $e_{k}$ is an orthonormal set such that infinitely many $e_{k}$ 's belong to $N^{\perp}$ for every $N<I$ in $\mathcal{N}$, then there is a partial isometry in $\mathcal{T}(\mathcal{N})$ such that $S S^{*}=P$ and its domain is spanned by a subset of the $e_{k}$ 's.

Let $e_{k}$ be an orthonormal set such that each $e_{k}=M_{k}^{\perp} e_{k}$ for some $M_{k} \in \mathcal{N}$ with $M_{k+}>0$ and infinitely many $e_{k}$ 's belong to $N^{\perp}$ for every $N<I$ in $\mathcal{N}$. Also let $f_{k}$ be an orthonormal set such that each $f_{k}=N_{k} f_{k}$ for some $N_{k} \in \mathcal{N}$ with $N_{k-}<I$ and infinitely many $f_{k}$ 's belong to $N$ for every $N>0$ in $\mathcal{N}$. Then there is a partial isometry in $\mathcal{T}(\mathcal{N})$ with initial space $\operatorname{span}\left\{e_{k}: k \geq 1\right\}$ and range space $\operatorname{span}\left\{f_{k}: k \geq 1\right\}$.

Proof. If $E=0_{+}$is an atom, then $\mathcal{F}=\left\{f_{k}: f_{k}=E f_{k}\right\}$ is infinite and thus $E$ is infinite rank. In this case, any partial isometry $S$ in $\mathcal{B}(\mathcal{H})$ with $S^{*} S=Q$ and range spanned by a subset of $\mathcal{F}$ will suffice. Otherwise, there is a sequence $N_{k}$ in $\mathcal{N}$ strictly decreasing with limit 0 . Choose an orthonormal basis for each $\left(N_{k}-N_{k+1}\right) Q \mathcal{H}$ starting with $N_{0}=I$, and combine them to form an orthonormal basis $e_{k}, k \geq 1$, for $Q \mathcal{H}$ with the property that each $e_{k}=M_{k}^{\perp} e_{k}$ for some $M_{k} \in \mathcal{N}$ with $M_{k}>0$. Then choose an increasing sequence $n_{k}$ such that $f_{n_{k}}$ is contained in $M_{k}$ for each $k \geq 1$. The operator $S=\sum_{k>1} f_{n_{k}} e_{k}^{*}$ does the job.

The second case is obtained from the first by passing to the orthogonal nest and taking adjoints.

In the last situation, the partial isometry $S$ is constructed using a well-known combinatorial device to determine an infinite permutation $\pi$ of $\mathbb{N}$ such that

$$
\operatorname{supp}\left(f_{\pi(k)}\right) \preceq \operatorname{supp}\left(e_{k}\right) \quad \text { for all } k \in \mathbb{N} .
$$

Indeed, at the $k$ th stage, if $\pi(k)$ is not defined, choose it to be an integer $n=: \pi(k)$ not yet in the range of $\pi$ such that (1) holds. This is possible since $e_{k}=M_{k}^{\perp} e_{k}$ and infinitely many $f_{n}$ 's are supported on $M_{k+}$. Then if $k$ is not yet in the range of $\pi$, choose $n=\pi^{-1}(k)$ in the same manner to be an integer on which $\pi$ is as yet undefined and for which $\operatorname{supp}\left(f_{k}\right) \preceq \operatorname{supp}\left(e_{n}\right)$ holds. In this way, a bijection satisfying (1) is obtained. Then defining $S=\sum_{k \geq 1} f_{\pi(k)} e_{k}^{*}$ yields the desired map.

Say that a nest $\mathcal{N}$ is admissible if both $0_{+}$and $I_{-}^{\perp}$ are either zero or infinite rank. In this case, it is easy to construct a projection $P=\sum_{-\infty}^{\infty} E_{n}$ in $\mathcal{N}^{\prime}$ such that each $E_{n}$ is an orthogonal projection in $\mathcal{N}^{\prime}, E_{n} \preceq E_{n+1}$ in the order on the nest (where we allow more than one of these projections to live in a single infinite atom to accommodate the case in which either $0_{+}$or $I_{-}^{\perp}$ is infinite rank) and the 
smallest interval of $\mathcal{N}$ containing $P$ is the identity $I$. Such a projection will also be called admissible. Note that $\mathcal{N}$ is admissible if and only if there are admissible projections in $\mathcal{T}(\mathcal{N})$. In the case of infinite multiplicity, this construction was used by Anoussis and Katsoulis [1], in which the $E_{n}$ are infinite rank. When both $P$ and $P^{\perp}$ had this property, they called it a sliced projection. The other case of interest occurs when there is an initial interval isomorphic to $(\omega+1)^{*}$ or a terminal interval isomorphic to $\omega+1$ with finite rank atoms. In this case, the $E_{n}$ must eventually be finite rank too. Note that any admissible projection $P$ may be decomposed as a sum of countably many pairwise orthogonal admissible projections by splitting $\mathbb{Z}$ into countably many disjoint subsets which are not bounded above or below.

Theorem 2. Let $\mathcal{N}$ be an admissible nest with admissible projections $P$ and $Q$. Suppose that $A$ is an element of $\mathcal{T}(\mathcal{N})$ such that $A=Q^{\perp} A P^{\perp}$ and $\|A\|<1$. Then there is a unitary $U$ in $\mathcal{T}(\mathcal{N})$ such that $Q^{\perp} U P^{\perp}=A$. Thus $A$ is the average of four unitaries in $\mathcal{T}(\mathcal{N})$.

Proof. Let $E_{n}$ be the subintervals of $P$ ordered by $\mathbb{Z}$ in the partial order on subintervals of $\mathcal{N}$; and choose a unit vector $e_{n}$ in the range of $E_{n}$ for $n \in \mathbb{Z}$. Similarly, let $F_{n}$ be the subintervals of $Q$ and let $f_{n}$ be unit vectors in $F_{n} \mathcal{H}$ for $n \in \mathbb{Z}$. Let $P_{0}$ and $P_{1}$ be the projections onto $\operatorname{span}\left\{e_{2 n}: n \in \mathbb{Z}\right\}$ and $\operatorname{span}\left\{e_{2 n+1}: n \in \mathbb{Z}\right\}$ respectively. Similarly define $Q_{0}$ and $Q_{1}$.

Since $A$ is a strict contraction, $P^{\perp}-A^{*} A$ and $Q^{\perp}-A A^{*}$ are positive and invertible in $\mathcal{B}\left(P^{\perp} \mathcal{H}\right)$ and $\mathcal{B}\left(Q^{\perp} \mathcal{H}\right)$ respectively. Thus by [4] (cf. [2, Corollary 14.5]), there are invertible operators $X$ in $\mathcal{T}\left(P^{\perp} \mathcal{N}\right)=P^{\perp} \mathcal{T}(\mathcal{N}) P^{\perp}$ and $Y$ in $\mathcal{T}\left(Q^{\perp} \mathcal{N}\right)=Q^{\perp} \mathcal{T}(\mathcal{N}) Q^{\perp}$ such that

$$
P^{\perp}-A^{*} A=X^{*} X \quad \text { and } \quad Q^{\perp}-A A^{*}=Y Y^{*} .
$$

(The inverses of $X$ and $Y$ need not belong to the nest algebra.) Let $U_{1}=P^{\perp} U_{1} P^{\perp}$ and $U_{2}=Q^{\perp} U_{2} Q^{\perp}$ be the partial isometries in $\mathcal{B}(\mathcal{H})$ such that

$$
X=U_{1}\left(P^{\perp}-A^{*} A\right)^{1 / 2} \text { and } Y=\left(Q^{\perp}-A A^{*}\right)^{1 / 2} U_{2} .
$$

Then setting $T=-U_{1} A^{*} U_{2}=P^{\perp} T Q^{\perp}$, we obtain a unitary operator $\left[\begin{array}{c}A \\ X\end{array}\right]$ ] from $P^{\perp} \mathcal{H} \oplus Q^{\perp} \mathcal{H}$ onto $Q^{\perp} \mathcal{H} \oplus P^{\perp} \mathcal{H}$.

To make use of this, fix a proper element $N$ in $\mathcal{N}$ and use Lemma 1 to construct a partial isometry $W_{1}$ in $\mathcal{T}(\mathcal{N})$ such that $W_{1}^{*} W_{1}=P^{\perp}$, with range contained in $N Q_{0} \mathcal{H}$ and spanned by a subset of the $f_{n}$ 's. Similarly construct another partial isometry $W_{2}$ in $\mathcal{T}(\mathcal{N})$ such that $W_{2} W_{2}^{*}=Q^{\perp}$, with domain contained in $N^{\perp} P_{0} \mathcal{H}$ spanned by a subset of the $e_{n}$ 's. Since $W_{1} T W_{2}$ has initial space contained in $N^{\perp} P \mathcal{H}$ and range in $N Q \mathcal{H}$, it belongs to $\mathcal{T}(\mathcal{N})$. Decomposing $\mathcal{B}(\mathcal{H})$ as operators from $\mathcal{H}=P^{\perp} \mathcal{H} \oplus P \mathcal{H}$ into $\mathcal{H}=Q^{\perp} \mathcal{H} \oplus Q \mathcal{H}$, we see that

$$
S=A+Y W_{2}+W_{1} X+W_{1} T W_{2} \simeq\left[\begin{array}{cc}
A & Y W_{2} \\
W_{1} X & W_{1} T W_{2}
\end{array}\right]
$$

is a partial isometry such that

$$
S^{*} S=P^{\perp}+W_{2}^{*} W_{2} \quad \text { and } \quad S S^{*}=Q^{\perp}+W_{1} W_{1}^{*} .
$$

The projection $I-S S^{*}$ dominates $P_{1}$ and is spanned by the properly supported projections $E_{n}$ or $E_{n}-e_{n} e_{n}^{*}$ for $n \in \mathbb{Z}$. Similarly, $I-S^{*} S$ dominates $Q_{1}$ and is spanned by the properly supported projections $F_{n}$ or $F_{n}-f_{n} f_{n}^{*}$ for $n \in \mathbb{Z}$. Thus they have infinite rank intersection with $N$ and $N^{\perp}$ for every $N \in \mathcal{N} \backslash\{0, I\}$, and their ranges are spanned by subspaces which have proper support. Therefore one 
may choose orthonormal bases for each of these subspaces to satisfy the hypothesis of Lemma 1. Hence there is a partial isometry $S_{1}$ in $\mathcal{T}(\mathcal{N})$ with initial projection $I-S^{*} S$ and range projection $I-S S^{*}$. Therefore $U=S+S_{1}$ is a unitary operator such that

$$
Q^{\perp} U P^{\perp}=Q^{\perp} S P^{\perp}=A .
$$

Finally, define unitary operators

$$
U_{k}=\left(Q^{\perp}+i^{k} Q\right) U\left(P^{\perp}+i^{k} P\right) \quad \text { for } \quad 1 \leq k \leq 4 .
$$

Compute:

$$
\frac{1}{4} \sum_{k=1}^{4} U_{k}=Q^{\perp} U P^{\perp}=A .
$$

The main result is now completed in a manner very similar to the methods of Anoussis and Katsoulis.

Theorem 3. If $\mathcal{N}$ is a nest such that $0_{+}$and $I_{-}^{\perp}$ have ranks either zero or infinity $\left(\mathcal{N}\right.$ is admissible), then every element $A \in \mathcal{T}(\mathcal{N})$ such that $\|A\| \leq 1-\frac{1}{n}$ is the average of $16 n^{2}$ unitary elements of $\mathcal{T}(\mathcal{N})$. Thus the convex hull of these unitary elements contains the whole open unit ball.

If $\mathcal{N}$ is not admissible, the wOT-closed convex hull of the unitary elements in $\mathcal{T}(\mathcal{N})$ is not the whole unit ball.

Proof. Let us establish the second part first. If $E=0_{+}$is a finite rank atom of $\mathcal{N}$, it is evident that for any unitary $U \in \mathcal{T}(\mathcal{N})$, one has $U E=E U$ and thus $E U E^{\perp}=0$. This persists in the WOT-closure of the convex hull; and thus it is not equal to the whole unit ball. A similar analysis works when $E=I-I_{-}$is a finite rank atom. Thus for a Russo-Dye theorem to be valid, it is necessary that $\mathcal{N}$ be admissible.

Let $P$ and $Q$ be admissible projections. Split $P$ and $Q$ into $2 n$ orthogonal admissible projections $P_{1}, \ldots, P_{2 n}$ and $Q_{1}, \ldots, Q_{2 n}$ respectively. It follows that $X_{i}=Q_{i}^{\perp}-\frac{1}{2 n} Q^{\perp}$ and $Y_{i}=P_{i}^{\perp}-\frac{1}{2 n} P^{\perp}$ are contractions in $\mathcal{N}^{\prime}$ such that

$$
\sum_{i=1}^{2 n} X_{i}=(2 n-1) I=\sum_{i=1}^{2 n} Y_{i} .
$$

Moreover, $\left(1-\frac{1}{2 n}\right)^{2}>1-\frac{1}{n}$. Thus the operators

$$
A_{i j}=\left(\frac{2 n}{2 n-1}\right)^{2} X_{i} A Y_{j}=Q_{i}^{\perp} A_{i j} P_{j}^{\perp} \quad \text { for } \quad 1 \leq i, j \leq n
$$

are strict contractions in $\mathcal{T}(\mathcal{N})$. By Theorem 2, each $A_{i j}$ is the average of four unitaries $U_{i j k}$ for $1 \leq k \leq 4$. Hence

$$
\begin{aligned}
\frac{1}{16 n^{2}} \sum_{i=1}^{2 n} \sum_{j=1}^{2 n} \sum_{k=1}^{4} U_{i j k} & =\frac{1}{4 n^{2}} \sum_{i=1}^{2 n} \sum_{j=1}^{2 n} A_{i j} \\
& =\frac{1}{4 n^{2}}\left(\sum_{i=1}^{2 n} X_{i}\right)\left(\frac{2 n}{2 n-1}\right)^{2} A\left(\sum_{j=1}^{2 n} Y_{j}\right) \\
& =\frac{1}{4 n^{2}}(2 n-1)^{2}\left(\frac{2 n}{2 n-1}\right)^{2} A=A .
\end{aligned}
$$




\section{REFERENCES}

1. M. Anoussis and E. Katsoulis, A nonself-adjoint Russo-Dye Theorem, Math. Ann. 304, (1996), 685-699. MR 97f:47042

2. K.R. Davidson, Nest Algebras, Pitman Research Notes in Mathematics Series, vol. 191, Longman Scientific and Technical Pub. Co., London, New York, 1988. MR 90f:47062

3. R.V. Kadison and G.K. Pedersen, Means and convex combinations of unitary operators, Math. Scand. 57 (1985), 245-266. MR 87g:47078

4. D.R. Larson, Nest algebras and similarity transformations, Ann. Math. 121 (1985), 409-427. MR 86j: 47061

5. R. Moore and T. Trent, Extreme points of certain operator algebras, Indiana U. Math. J. 36 (1987), 645-650. MR 89d:47103

6. B. Russo and H. Dye, A note on unitary operators in $C^{*}$-algebras, Duke Math. J. 33 (1966), 413-416. MR 33:1750

Department of Pure Mathematics, University of Waterloo, Waterloo, Ontario, CANADA N2L 3G1

E-mail address: krdavidson@math.uwaterloo.ca 